\title{
The Lymphatic Infiltration Identified by D2-40 Monoclonal Antibody Predicts Lymph Node Metastasis in Submucosal Invasive Colorectal Cancer
}

\author{
Ken Kawaura a, e Satoshi Fujii ${ }^{a}$ Yukinori Murata ${ }^{b}$ Takahiro Hasebe ${ }^{a}$ \\ Genichiro Ishii $^{\text {a }}$ Tohru Itoh $^{e}$ Yasushi Sano $^{c}$ Norio Saito $^{d}$ Atsushi Ochiai $^{a}$ \\ a Pathology Division, National Cancer Center Research Institute East and ${ }^{b}$ Clinical Laboratory Division, \\ 'Endoscopy and Gastrointestinal Oncology and d Colorectal (Pelvic) Surgery, National Cancer Center Hospital East, \\ Kashiwa, Chiba; ${ }^{e}$ Endoscopy Division, Kanazawa Medical University, Kanazawa, Ishikawa, Japan
}

\begin{abstract}
Key Words
Colorectal cancer, submucosal invasive $\cdot$ Lymph node metastasis $\cdot$ Lymphatic infiltration $\cdot$ D2-40 - Risk factor • Endoscopic resection
\end{abstract}

\begin{abstract}
Background and Study Aims: Lymphatic infiltration has been recognized as a significant risk factor for lymph node metastasis of submucosal invasive colorectal cancer (SICC), but it is difficult to detect microscopically on hematoxylin and eosin (H\&E)-stained slides. We therefore identified lymphatic infiltration of tumor cells with D2-40 monoclonal antibody, which reacts specifically against the endothelium of lymphatic vessels, to make an objective and precise diagnosis. Patients and Methods: The surgical specimens of 122 consecutive patients with nonpedunculated SICC were examined for lymphatic infiltration by immunohistochemical staining with D2-40 monoclonal antibody (LI-D) and for venous infiltration by Elastica van Gieson staining (VI-E). $R \boldsymbol{e}$ sults: Lymph node metastasis was found in 20 patients. Multivariate analysis showed that LI-D $(p=0.0415)$ and VI-E $(p=$ 0.0119 ) were significant risk factors for lymph node metastasis. Regardless of the presence of risk factors including at least either lymphatic infiltration or venous infiltration, no lymph node metastasis-positive patients were found (0\%) among the 25 patients whose colorectal cancer had a sub-
\end{abstract}

mucosal invasive depth of less than 1,500 $\mu \mathrm{m}$. No lymph node metastasis was found in any of the patients with a depth of submucosal invasion of less than $3,000 \mu \mathrm{m}$, who had no risk factors, including LI-D or VI-E. Conclusions: Correct evaluation of lymphatic infiltration by immunohistochemical staining with D2-40 monoclonal antibody may play a crucial role in determining whether there are indications for additional treatment in the management of endoscopically resected SICC.

Copyright $\odot 2007$ S. Karger AG, Basel

\section{Introduction}

The rate of detection of colorectal cancer in the early stage has increased in recent years as a result of improvements in endoscopic technology [1-3] and for this reason the number of endoscopists and pathologists has increased to examine submucosal invasive colorectal cancers (SICCs) that are endoscopically resected [4-6]. Complete endoscopic resection (ER) of intramucosal carcinoma is regarded as curative treatment since such lesions have been found to be associated with almost no risk of lymph node metastasis. Conversely, it has been reported that $10-16 \%$ of SICC patients have lymph node metastasis [7-15]. Most endoscopists have recognized that patients who may have lymph node metastasis based on the results 
of pathological examination of endoscopically resected specimens generally require additional surgical resection accompanied by regional lymph node dissection as a curative treatment. However, since thus far there have been no universally accepted indications for additional surgical resection, those patients found to have no metastases by final diagnosis of the lymph nodes dissected had also undergone additional surgical resection of the colorectum. As stated above, maximally $80-90 \%$ of SICC patients are estimated to have undergone unnecessary surgical resection.

Thus, accurate indications for additional and inevitable surgical resection of the colorectum need to be established to avoid local recurrence and extended metastasis after ER. Some pathological risk factors, including depth of submucosal invasion [7, 9, 15-18], histological differentiation $[7,8,10,11,14,19]$, venous infiltration [13, $14]$, and lymphatic infiltration $[10,12-15,20]$, have been evaluated as candidates for risk factors that predict lymph node metastasis of SICC.

Venous infiltration has been precisely evaluated by Elastica van Gieson staining [21] but only hematoxylin and eosin (H\&E)-stained slides have been routinely used to detect lymphatic infiltration. The recent use of D2-40 monoclonal antibody, which specifically reacts with the endothelium of lymphatic vessels [22-28], has made it possible to identify lymphatic infiltration objectively. In fact, the identification of lymphatic infiltration with D240 monoclonal antibody and the clinicopathological implications of lymphatic infiltration have been reported in cancer of the breast [29], tonsil [23], and head and neck [22]. However, since no similar studies have been performed in gastrointestinal cancers, we used immunohistochemical staining with D2-40 monoclonal antibody to examine SICCs for the presence of lymphatic infiltration and the relation between its presence and lymph node metastasis.

In this study we examined surgical specimens of SICC for lymphatic infiltration by immunohistochemical staining with D2-40 monoclonal antibody (LI-D) and for venous infiltration by Elastica van Gieson staining (VIE). We also analyzed the relationship between positivity for these two factors and lymph node metastasis in SICC.

In addition, we investigated whether accurate identification of vessel infiltration by tumor cells correlated with the presence of lymph node metastasis and could serve as an indication for the necessity of additional surgical resection in SICC.

Real Risk Factors for Lymph Node Metastases

\section{Patients and Methods}

\section{Patients}

The specimens of 122 consecutive patients with SICC without a stalk (nonpedunculated cancer) surgically treated between July 1992 and March 2005 at the National Cancer Center Hospital East were collected for use in the present study. Patients who had received chemotherapy or radiation therapy before surgery were excluded. Patients with familial adenomatous polyposis and synchronous colorectal cancers and with synchronous colorectal cancers secondary to inflammatory bowel disease were also excluded. Clinical information was obtained from the patients' medical records. The patients included 77 men and 45 women, and their average age at the time of surgery was 62.1 years (range 38-84 years). The cancers in this study were classified according to locus into 79 colon cancers and 43 rectal cancers. A total of 92 patients (75.4\%) underwent surgical resection plus regional lymph node dissection and 30 patients (24.6\%) underwent ER followed by surgical resection plus regional lymph node dissection. Lymph node metastasis was found in 20 of the 122 patients (16.4\%). The nonpedunculated tumors were macroscopically classified into two subtypes, the depressed type and the nondepressed type, according to the criteria of the Japanese Society for Cancer of the Colon and Rectum (JSCCR). This study was performed in accordance with the Helsinki Declaration and informed consent was obtained from all the patients whose specimens were examined.

\section{Pathological Examination}

For routine pathological examination, the surgically resected specimens were fixed in $10 \%$ formalin at room temperature, and the entire tumor was cut into about 2- to 4 -mm-thick sections to prepare slides for microscopic analysis. All specimens cut were embedded in paraffin, and tissue sections of each tumor were cut at a thickness of $4 \mu \mathrm{m}$. We selected a representative section with the maximum diameter of each tumor containing cancerous tissue at the deepest portion for the analyses of several clinicopathological parameters of all tissue sections prepared. One slice of the selected section was stained with $\mathrm{H} \& \mathrm{E}$ for routine pathological diagnosis. One slice of the same section was used for Elastica van Gieson staining to examine the tumor tissue for venous infiltration by tumor cells, and another slice was used for immunohistochemical staining with D2-40 monoclonal antibody to detect lymphatic infiltration by tumor cells. The tissue was simultaneously examined for the following pathological parameters: tumor size, the level of depth of submucosal invasion, main differential subtype, differential subtype at the deepest portion, coexistence of an adenomatous component, inflammatory reaction around the cancerous tissue, and tumor budding. The differential subtype was classified according to the World Health Organization classification.

\section{Method of Measuring Depth of Submucosal Invasion Microscopically}

When it was possible to identify the lamina muscularis mucosae as the baseline by H\&E staining, the vertical distance from the baseline to the site of deepest invasion was recorded as the depth of submucosal invasion [15]. When the lamina muscularis mucosae had been destroyed by carcinomatous invasion, the superficial aspect of tumor was used as the baseline, and the vertical distance from the baseline to the site of deepest invasion was recorded as the depth of submucosal invasion [15]. 


\section{Definition of Tumor Budding}

A focus of tumor budding was defined as an isolated single cancer cell or a small cluster of less than four cancerous cells in the invasive area of the tumor [30-32]. Scattered foci are usually observed in the stroma of the invasive frontal region. Cancerous foci in portions of the tumor where it was fragmented by inflammatory cell aggregation or technical artifacts were not identified as tumor budding. After selecting the field in which 'budding' intensity appeared to be maximal (in the sense that budding foci were easily identified), the number of tumor budding foci in a single field observed through a $20 \times$ objective lens was counted. When the number was no more than four per field (0-4) budding was classified as low grade for tumor budding, and when 5 or more were present (5), it was classified as high grade [30].

\section{Immunohistochemical Analysis with D2-40 Monoclonal} Antibody

Paraffin-embedded slices on silane-coated slides were dewaxed and dehydrated. After inactivating endogenous peroxidase with 3\% hydrogen peroxidase for $20 \mathrm{~min}$, the slices were heated in citrate buffer ( $\mathrm{pH} 6.0$ ) in a microwave oven at $95^{\circ} \mathrm{C}$ for $20 \mathrm{~min}$ for antigen retrieval. Thin slices put on slides were incubated with the D2-40 monoclonal antibody (1:200 dilution, Signet Laboratories, Dedham, Mass., USA) at $4^{\circ} \mathrm{C}$ overnight. The D2-40 monoclonal antibody was generated against an oncofetal antigen expressed in fetal testis and testicular germ cell tumors and has been found to also react with differentiated lymphatic endothelium, but not with arterial or venous endothelium. Immunohistochemical staining was performed for 3 min with the EnVision and HRP DAB system (DAKO Cytomation, Carpinteria, Calif., USA), and the nuclei were stained with hematoxylin.

Assessment of Lymphatic Infiltration by Immunohistochemical Staining with D2-40 Monoclonal Antibody

In this study 'lymphatic infiltration' was defined as a tumor embolus in a vessel lined with lymphatic endothelium that stained immunohistochemically with D2-40 monoclonal antibody (fig. 1). We investigated the density of lymphatic vessels in the densely vascularized area at the invasive front of the tumor, and counted the number of lymphatic vessels that stained immunohistochemically with D2-40 monoclonal antibody in a high-power field $(\times 200)$. The numbers of lymphatic vessels in each of three areas were added and divided by three to obtain the average number; the average number was recorded as the density of the lymphatic vessel.

\section{Statistical Analysis}

Statistical analyses of differences between the two groups were performed by the Mann-Whitney U test or Fisher's exact test. The relationships between several clinicopathological factors and the factor 'lymph node metastasis' were investigated by univariate and multivariate analyses with logistic regression analysis. $\mathrm{p}<$ 0.05 was defined as statistically significant in this study.

\section{Results}

\section{Detection of Lymphatic Infiltration}

Table 1 shows the immunohistochemical staining with D2-40 monoclonal antibody and H\&E staining for the identification of lymphatic infiltration associated with lymph node metastases. The tumors of 18 (14.8\%) of the 122 patients were found to be positive for lymphatic infiltration by H\&E staining, and the number rises to 42 (34.4\%) when examined by immunohistochemical staining with D2-40 antibody. There were 14 patients (70\%) with both lymph node metastases and LI-D in comparison with 7 (35\%) with H\&E staining (table 1). Consequently immunohistochemical staining with D2-40 monoclonal antibody picked up 7 more patients who had not been detected by H\&E staining ( $<<0.001, \chi^{2}$ test).

\section{Relationship between Lymph Node Metastasis and Several Clinicopathological Factors}

Table 2 shows the relationship between lymph node metastasis and clinicopathological factors in the present study. Lymph node metastasis-positive patients tended to have deeper tumor invasion than negative patients $(\mathrm{p}=$ $0.0109)$. LI-D was observed in 42 patients (34.4\%). There were $14(14 / 20,70.0 \%)$ lymph node metastasis-positive patients with LI-D and $6(6 / 20,30.0 \%)$ without LI-D. VIE was observed in 33 patients (27.0\%). There were 12 lymph node metastasis-positive patients with venous infiltration $(12 / 20,60.0 \%)$, and 8 lymph node metastasispositive patients without venous infiltration $(8 / 20,40.0 \%)$. Differential subtype ( $\mathrm{p}=0.0017), \mathrm{LI}-\mathrm{D}(\mathrm{p}=0.0005)$, VI-E $(\mathrm{p}=0.0007)$, and high-grade tumor budding $(\mathrm{p}=0.0238)$ were significantly associated with the presence of lymph node metastasis according to the results of the univariate analysis (table 2).

\section{Multivariate Analysis of Risk Factors for Lymph Node Metastasis}

In analyzing the relationship between the submucosal invasive depth and lymph node metastasis, we tried to choose several setting points in performing univariate analyses. Since $2,000 \mu \mathrm{m}$ showed the highest odds ratio, we chose the setting point of $2,000 \mu \mathrm{m}$.

There was also a significant correlation between lymphatic infiltration with H\&E staining and lymph node metastasis, showing that the odds ratio was 4.455 and the p value was 0.0084 ; however, the odds ratio was 6.167 and the $p$ value was 0.0007 in univariate analysis by evaluating lymphatic infiltration with D2-40 antibody in relation to lymph node metastasis. We concluded that the evaluation of lymphatic infiltration using immunohistochemical staining with D2-40 antibody was more sensitive to examine risk factors for lymph node metastasis and used multivariate analysis with the data of lymphatic infiltration with D2-40 antibody. 
Fig. 1. Representative photographs of tumor emboli in lymphatic vessel stained immunohistochemically with D2-40 monoclonal antibody are shown. Photographs of $\mathrm{H} \& \mathrm{E}$ staining (a, c, e, g) correspond with immunohistochemical staining with D2-40 antibody (b, d, f, h). a, b D2-40 monoclonal antibody immunohistochemically reacts with lymphatic vessels, whereas the adjacent blood vessels are negative for immunohistochemical staining with D2-40 monoclonal antibody. c, d It is difficult to recognize lymphatic infiltration by $\mathrm{H} \& \mathrm{E}$ staining, but we can clearly recognize the lymphatic infiltration by immunohistochemical staining with D2-40 monoclonal antibody. e, f Positive immunohistochemical staining with the D2-40 antibody is seen in microlymphatic vessels including small tumor emboli. $\mathbf{g}, \mathbf{h}$ By immunohistochemical staining using D2-40 antibody, lymphatic vessels including emboli of cancer cells were discriminated from spaces around nests of cancer cells generated by retraction artifact.
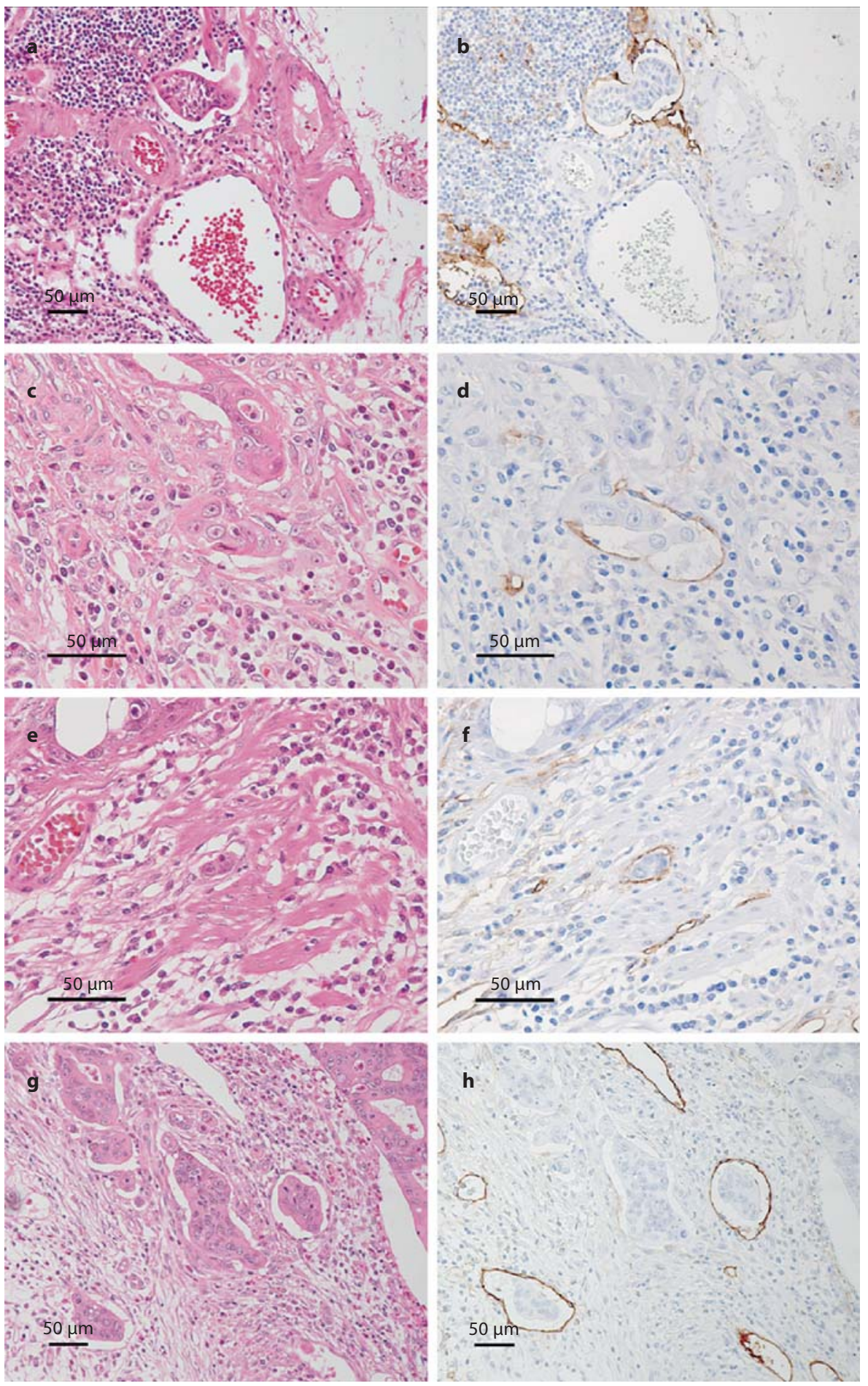

According to the multivariate analysis, LI-D ( $\mathrm{p}=$ $0.0415)$ and VI-E ( $\mathrm{p}=0.0119)$ were selected as significant risk factors for lymph node metastasis (table 3), suggesting that patients who were not positive for lymphatic in- filtration by immunohistochemical staining with D2-40 monoclonal antibody or venous infiltration constituted a risk factor-negative group for lymph node metastasis. Conversely, patients with lymphatic infiltration or ve- 
nous infiltration may constitute a risk factor-positive group for lymph node metastasis.

\section{Relationship between Risk Factors, Including}

Lymphatic Infiltration or Venous Infiltration, and

Lymph Node Metastasis according to Depth of

\section{Submucosal Invasion}

The results of the analyses described above suggested that both LI-D and VI-E are risk factors for SICC.

Regardless of the presence of risk factors, including at least either lymphatic infiltration or venous infiltration, no $(0 \%)$ lymph node metastasis-positive patients were among the 25 patients whose colorectal cancers were found to have a submucosal invasive depth less than 1,500 $\mu \mathrm{m}$. None of the patients whose colorectal cancers were found to have a submucosal invasive depth less than 3,000 $\mu \mathrm{m}$, who had no risk factors, including LI-D or VI-E, had no lymph node metastasis. In addition, only 1 patient was detected even among the patients whose colorectal cancers had a submucosal invasive depth less than $5,000 \mu \mathrm{m}$ and neither LI-D nor VI-E (table 4).

Table 1. Comparison between immunohistochemical staining with D2-40 monoclonal antibody and $\mathrm{H} \& \mathrm{E}$ staining for detection of lymphatic infiltration

\begin{tabular}{llc}
\hline & $\begin{array}{l}\text { Lymphatic infiltration- } \\
\text { positive }\end{array}$ & $\begin{array}{l}\text { LNM with lymphatic } \\
\text { infiltration }\end{array}$ \\
\hline H\&E & $18 / 122(14.8 \%)$ & $7 / 122(5.7 \%)$ \\
D2-40 & $42 / 122(34.4 \%)$ & $14 / 122(11.5 \%)$ \\
\hline
\end{tabular}

$\chi^{2}$ test $\mathrm{p}<0.001 . \mathrm{LNM}=\mathrm{Lymph}$ node metastasis-positive patients. Total number of patients: 122 .

Table 2. Relationship between lymph node metastasis and clinicopathological factors

\begin{tabular}{|c|c|c|c|c|c|}
\hline \multirow[t]{2}{*}{ Variable } & & \multirow[t]{2}{*}{ Patients } & \multicolumn{2}{|c|}{ Lymph node metastasis } & \multirow[t]{2}{*}{$\mathrm{p}$ value } \\
\hline & & & negative $(n=102)$ & positive $(n=20)$ & \\
\hline \multirow[t]{2}{*}{ Location } & Colon & 79 & $68(86.1)$ & $11(13.9)$ & 0.3200 \\
\hline & Rectum & 43 & $34(79.1)$ & $9(20.9)$ & \\
\hline Macroscopic type & Depressed type & 78 & $64(82.1)$ & $14(17.9)$ & 0.6175 \\
\hline \multirow[t]{3}{*}{ Differential subtype } & Well & 57 & $52(91.2)$ & $5(8.8)$ & 0.0017 \\
\hline & Moderate & 61 & $49(80.3)$ & $12(19.7)$ & \\
\hline & Poor & 4 & $1(25.0)$ & $3(75.0)$ & \\
\hline \multirow[t]{2}{*}{ Differential subtype at the deepest portion } & Well & 40 & $36(90.0)$ & $4(10.0)$ & 0.2061 \\
\hline & Moderate or poor & 82 & $66(80.5)$ & $16(19.5)$ & \\
\hline \multirow[t]{2}{*}{ Density of lymph vessels ${ }^{\mathrm{d}}$} & $\leq 13$ & 67 & $55(82.1)$ & $12(17.9)$ & 0.8065 \\
\hline & $>13$ & 55 & $47(85.5)$ & $8(14.5)$ & \\
\hline \multirow[t]{2}{*}{ Venous infiltration $^{\mathrm{e}}$} & Absent & 89 & $81(91.0)$ & $8(9.0)$ & 0.0007 \\
\hline & Present & 33 & $21(63.6)$ & $12(36.4)$ & \\
\hline \multirow[t]{2}{*}{ Adenomatous component } & Absent & 79 & $68(86.1)$ & $11(13.9)$ & 0.3200 \\
\hline & Present & 43 & $34(79.1)$ & $9(20.9)$ & \\
\hline \multirow[t]{2}{*}{ Inflammatory reaction } & Absent & 80 & $65(81.3)$ & $15(18.8)$ & 0.4428 \\
\hline & Present & 42 & $37(88.1)$ & $5(11.9)$ & \\
\hline \multirow[t]{2}{*}{ Tumor budding } & Low grade $(0-4)$ & 99 & $87(87.9)$ & $12(12.1)$ & 0.0238 \\
\hline & High grade $(5+)$ & 23 & $15(65.2)$ & $8(34.8)$ & \\
\hline
\end{tabular}

${ }^{a}$ Fisher's exact test. ${ }^{\mathrm{b}}$ Mann-Whitney U test. ${ }^{\mathrm{c}}$ Lymphatic infiltration by immunohistochemical staining with D2-40 monoclonal antibody. ${ }^{\mathrm{d}}$ Average of number of lymph vessels. ${ }^{\mathrm{e}}$ Venous infiltration by Elastica van Gieson staining. Figures in parentheses represent percentage. 
Table 3. Univariate or multivariate analysis of five factors in relation to lymph node metastasis

\begin{tabular}{|c|c|c|c|c|c|}
\hline Variable & & \multicolumn{2}{|l|}{ Univariate analysis } & \multicolumn{2}{|l|}{ Multivariate analysis } \\
\hline Lymphatic infiltration with H\&E staining & $\begin{array}{l}\text { Absent } \\
\text { Present }\end{array}$ & $\begin{array}{l}\text { referent } \\
4.455(1.465-13.540)\end{array}$ & 0.0084 & - & - \\
\hline Venous infiltration ${ }^{\mathrm{b}}$ & $\begin{array}{l}\text { Absent } \\
\text { Present }\end{array}$ & $\begin{array}{l}\text { referent } \\
5.786(2.096-15.970)\end{array}$ & 0.0007 & $\begin{array}{l}\text { referent } \\
4.614(1.401-15.190)\end{array}$ & 0.0119 \\
\hline Differential subtype & $\begin{array}{l}\text { Well } \\
\text { Moderate } \\
\text { Poor }\end{array}$ & $\begin{array}{l}\text { referent } \\
3.120(1.055-9.225) \\
17.824(1.750-181.546)\end{array}$ & $\begin{array}{l}0.0397 \\
0.0150\end{array}$ & $\begin{array}{l}\text { referent } \\
1.726(0.503-5.916) \\
5.373(0.311-92.734)\end{array}$ & $\begin{array}{l}0.3853 \\
0.2427\end{array}$ \\
\hline Tumor budding & $\begin{array}{l}\text { Low } \\
\text { High }\end{array}$ & $\begin{array}{l}\text { referent } \\
3.867(1.354-11.040)\end{array}$ & 0.0115 & $\begin{array}{l}\text { referent } \\
1.991(0.503-7.722)\end{array}$ & 0.3302 \\
\hline
\end{tabular}

$\mathrm{OR}=$ Odds ratio; $\mathrm{CI}=$ confidence intervals.

${ }^{\mathrm{a}}$ Lymphatic infiltration by immunohistochemical staining with D2-40 monoclonal antibody. ${ }^{\mathrm{b}}$ Venous infiltration by Elastica van Gieson staining.

Table 4. Relationship between risk factors and lymph node metastasis according to the depth of submucosal invasion

\begin{tabular}{lrrll}
\hline $\begin{array}{l}\text { Submucosal } \\
\text { depth, } \mu \mathrm{m}\end{array}$ & Patients & LNM & $\begin{array}{l}\text { LNM/risk factor- } \\
\text { negative patients }^{\mathrm{a}}\end{array}$ & $\begin{array}{l}\text { LNM/risk factor- } \\
\text { positive patients }^{\mathrm{b}}\end{array}$ \\
\hline $0<\mathrm{x}<500$ & 7 & 0 & $0 / 7$ & $0 / 0$ \\
$500 \leq \mathrm{x}<1,000$ & 8 & 0 & $0 / 5$ & $0 / 3$ \\
$1,000 \leq \mathrm{x}<1,500$ & 10 & 0 & $0 / 7$ & $0 / 3$ \\
$1,500 \leq \mathrm{x}<2,000$ & 12 & 1 & $0 / 7$ & $1 / 5$ \\
$2,000 \leq \mathrm{x}<2,500$ & 10 & 2 & $0 / 5$ & $2 / 5$ \\
$2,500 \leq \mathrm{x}<3,000$ & 10 & 1 & $0 / 6$ & $1 / 4$ \\
$3,000 \leq \mathrm{x}<3,500$ & 14 & 4 & $1 / 7$ & $3 / 7$ \\
$3,500 \leq \mathrm{x}<4,000$ & 7 & 1 & $0 / 4$ & $1 / 3$ \\
$4,000 \leq \mathrm{x}<4,500$ & 9 & 2 & $0 / 1$ & $2 / 8$ \\
$4,500 \leq \mathrm{x}<5,000$ & 8 & 3 & $0 / 1$ & $3 / 7$ \\
$5,000 \leq \mathrm{x}$ & 27 & 6 & $2 / 12$ & $4 / 15$ \\
\hline Total & 122 & 20 & $3 / 62(4.8 \%)$ & $17 / 60$ \\
& & & & $(28.3 \%)$ \\
\hline
\end{tabular}

LNM = Lymph node metastasis-positive patients.

${ }^{a}$ Patients with neither lymphatic infiltration by immunohistochemical staining with D2-40 monoclonal antibody nor venous infiltration by Elastica van Gieson staining.

${ }^{b}$ Patients with either lymphatic infiltration by immunohistochemical staining with D2-40 monoclonal antibody or venous infiltration by Elastica van Gieson staining.
SICC was divided into two groups for evaluating unnecessary additional treatment. One group consists of SICC with an invasive depth less than $1,500 \mu \mathrm{m}$ and no lymph node metastases, and the other consists of the SICCs with an invasive depths less than 3,000 $\mu \mathrm{m}$ and no risk factors, including LI-D or VI-E.

\section{Discussion}

There have been various reports on risk factors for lymph node metastasis in SICC [7, 10-15, 17, 33-35], but the management of patients with nonpedunculated SICC remains a matter of controversy. In a preliminary study of 122 patients surgically treated in our institution, the multivariate analysis selected the presence of lymphatic infiltration detected by H\&E staining and the presence of VI$\mathrm{E}$ as independent risk factors for lymph node metastasis in SICC. We evaluated the presence of VI-E in all patients as routine pathological diagnosis, which can be presumed to be accurate. Lymphatic infiltration in H\&E-stained sections is identified as tumor emboli in channels clearly lined by a single layer of endothelial cells [24]. However, we frequently encounter technical pitfalls in attempting to identify lymphatic vessel walls by H\&E staining. There are at least two pitfalls in regard to identifying tumor emboli. One pitfall is the impossibility of visualizing tumor emboli when the lumen of a lymphatic vessel is obliterated 
and the wall of a lymphatic vessel has been destroyed by the aggregation of tumor cells. The other pitfall is the impossibility of distinguishing retraction artifacts that isolate aggregates of tumor cells as a result of tissue shrinkage during fixation from true tumor emboli floating in lymphovascular spaces. To decrease the inaccuracy based on these obscure evaluations by $\mathrm{H} \& \mathrm{E}$ staining, we evaluated LI-D clearly. By doing so, we were able to more selectively identify a group with a low likelihood of lymph node metastasis that could be followed up by ER alone.

As shown in table 1, 42 lesions with LI-D were identified, whereas lymphatic infiltration was detected in only 18 cases by H\&E staining. The cases that were positive for lymphatic infiltration by $\mathrm{H} \& \mathrm{E}$ staining were found to actually be negative for LI-D, and thus were false-positive. On the other hand, 27 cases with no lymphatic infiltration by $\mathrm{H} \& \mathrm{E}$ staining were found to be positive for LI-D, and thus were false-negative. Several reasons for the results of the examinations by H\&E staining and immunohistochemical staining with D2-40 monoclonal antibody can be pointed out. Tumor emboli in microlymphatic vessels are sometimes difficult to detect by H\&E staining because they are in invasive areas with a strong host reaction, such as fibrocollagenous tissue proliferation. Spaces around nests of tumor cells that are caused by inevitable retraction of tissue shrinkage artifacts after tissue fixation may be confusing if true tumor emboli are floating in lymphovascular spaces. D2-40 monoclonal antibody, which reacts with the endothelium of differentiated lymphatic vessels alone, was very useful in overcoming the inaccuracy in identifying true tumor in lymphatic vessels.

Based on the results of this study, we propose that SICC be divided into two groups for the management of additional treatment. The following two groups were supposed not to have lymph node metastases: (1) SICCs with a submucosal invasive depth of less than $1,500 \mu \mathrm{m}$ $[13,15]$, and (2) SICCs with a submucosal invasive depth less than 3,000 $\mu \mathrm{m}$ and negative for risk factors, including LI-D and VI-E. A third subgroup, consisting of cancers with submucosal invasive depth less than 5,000 $\mu \mathrm{m}$ and negative for risk factors, including lymphatic infiltration and venous infiltration, might also be proposed for management. Only 1 (1.8\%) of the 56 patients who would have been in the third group in this study was found to have lymph node metastasis. Thus, we think the third group has a much lower risk of lymph node metastasis regardless of the depth of cancer invasion, if neither LI-D nor VI-E is detected at all. However, further study, including more patients, are necessary in the future to confirm the above grouping.
Since SICCs within a submucosal invasive depth of $5,000 \mu \mathrm{m}$ include almost all submucosal invasive nonpedunculated colorectal cancers that can be resected endoscopically, accurately examining for LI-D and VI-E might play an important role in identifying patients who can be followed up after ER, regardless of the submucosal depth of invasion. There are several methods of measuring submucosal invasive depth depending on whether the lamina muscularis mucosae is intact or has been destroyed. However, as stated above, we think that valid evaluation of lymphatic and venous infiltration may be of greater significance for the management of cases resected endoscopically.

We only evaluated the section with the maximum diameter and the most deeply invading portion of the tumor for immunohistochemical staining with D2-40 antibody to determine whether risk factors were present in our study; however, since we were able to detect risk factors in more patients than by H\&E staining, we think that our procedure is reliable on the validity of evaluation.

We excluded the pedunculated type of SICCs in our study, because the methods of measuring submucosal invasion in such lesions vary, and none has been established. Analysis such as our current study for pedunculated lesions will be also necessary since more pedunculated SICC were resected by ER.

The results of our study showed that both the results of the evaluation of lymphatic infiltration by immunohistochemical analysis with D2-40 antibody and of evaluation of venous infiltration by Elastica van Gieson staining were significantly correlated with the presence of lymph node metastasis. These analyses could represent real and practical indications in the management of SICC operated with the method of ER.

\section{Acknowledgments}

The authors wish to thank M. Okumoto for her technical assistance, and M. Suzaki and K. Sato for their secretarial assistance.

References

Kawaura/Fujii/Murata/Hasebe/Ishii/ Itoh/Sano/Saito/Ochiai 
3 Kaihara T, Sano Y, Okuno T, et al: Endoscopic therapy for colorectal cancer. Nippon Rinsho 2003;61(suppl 7):533-539.

4 Chantereau MJ, Faivre J, Boutron MC, et al: Epidemiology, management, and prognosis of malignant large bowel polyps within a defined population. Gut 1992;33:259-263.

5 Mainprize KS, Mortensen NJ, Warren BF: Early colorectal cancer: recognition, classification and treatment. Br J Surg 1998;85:469476.

-6 Akasu T, Kondo H, Moriya Y, et al: Endorectal ultrasonography and treatment of early stage rectal cancer. World J Surg 2000;24 1061-1068

7 Haggitt RC, Glotzbach RE, Soffer EE, Wruble LD: Prognostic factors in colorectal carcinomas arising in adenomas: implications for lesions removed by endoscopic polypectomy. Gastroenterology 1985;89:328-336.

-8 Coverlizza S, Risio M, Ferrari A, FenoglioPreiser CM, Rossini FP: Colorectal adenomas containing invasive carcinoma. Pathologic assessment of lymph node metastatic potential. Cancer 1989;64:1937-1947.

-9 Tanaka S, Yokota T, Saito D, Okamoto S, Oguro Y, Yoshida S: Clinicopathologic features of early rectal carcinoma and indications for endoscopic treatment. Dis Colon Rectum 1995;38:959-963.

10 Cooper HS, Deppisch LM, Gourley WK, et al: Endoscopically removed malignant colorectal polyps: clinicopathologic correlations. Gastroenterology 1995; 108: 16571665.

-11 Volk EE, Goldblum JR, Petras RE, Carey WD, Fazio VW: Management and outcome of patients with invasive carcinoma arising in colorectal polyps. Gastroenterology 1995; 109:1801-1807.

12 Netzer P, Forster C, Biral R, et al: Risk factor assessment of endoscopically removed malignant colorectal polyps. Gut 1998;43:669674.

13 Egashira Y, Yoshida T, Hirata I, et al: Analysis of pathological risk factors for lymph node metastasis of submucosal invasive colon cancer. Mod Pathol 2004;17:503-511.

14 Ueno H, Mochizuki H, Hashiguchi Y, et al: Risk factors for an adverse outcome in early invasive colorectal carcinoma. Gastroenterology 2004;127:385-394.
15 Kitajima K, Fujimori T, Fujii S, et al: Correlations between lymph node metastasis and depth of submucosal invasion in submucosal invasive colorectal carcinoma: a Japanese collaborative study. J Gastroenterol 2004;39: 534-543.

16 Nivatvongs S, Rojanasakul A, Reiman HM, et al: The risk of lymph node metastasis in colorectal polyps with invasive adenocarcinoma. Dis Colon Rectum 1991;34:323-328.

$\checkmark 17$ Kyzer S, Begin LR, Gordon PH, Mitmaker B: The care of patients with colorectal polyps that contain invasive adenocarcinoma. Endoscopic polypectomy or colectomy? Cancer 1992; 70:2044-2050.

18 Kudo S: Endoscopic mucosal resection of flat and depressed types of early colorectal cancer. Endoscopy 1993;25:455-461.

19 Wilcox GM, Anderson PB, Colacchio TA: Early invasive carcinoma in colonic polyps. A review of the literature with emphasis on the assessment of the risk of metastasis. Cancer 1986;57:160-171.

20 Muller S, Chesner IM, Egan MJ, et al: Significance of venous and lymphatic invasion in malignant polyps of the colon and rectum. Gut 1989;30:1385-1391.

21 Vass DG, Ainsworth R, Anderson JH, Murray $D$, Foulis $A K$ : The value of an elastic tissue stain in detecting venous invasion in colorectal cancer. J Clin Pathol 2004;57:769772.

22 Franchi A, Gallo O, Massi D, Baroni G, Santucci M: Tumor lymphangiogenesis in head and neck squamous cell carcinoma: a morphometric study with clinical correlations. Cancer 2004;101:973-978.

23 Kahn HJ, Bailey D, Marks A: Monoclonal antibody D2-40, a new marker of lymphatic endothelium, reacts with Kaposi's sarcoma and a subset of angiosarcomas. Mod Pathol 2002;15:434-440.

24 Kahn HJ, Marks A: A new monoclonal antibody, D2-40, for detection of lymphatic invasion in primary tumors. Lab Invest 2002; 82:1255-1257.

25 Dumoff KL, Chu C, Xu X, Pasha T, Zhang PJ, Acs G: Low D2-40 immunoreactivity correlates with lymphatic invasion and nodal metastasis in early-stage squamous cell carcinoma of the uterine cervix. Mod Pathol 2005; 18:97-104.
26 Fogt F, Pascha TL, Zhang PJ, Gausas RE, Rahemtulla A, Zimmerman RL: Proliferation of D2-40-expressing intestinal lymphatic vessels in the lamina propria in inflammatory bowel disease. Int J Mol Med 2004;13: 211-214.

27 Fogt F, Zimmerman RL, Ross HM, Daly T, Gausas RE: Identification of lymphatic vessels in malignant, adenomatous and normal colonic mucosa using the novel immunostain D2-40. Oncol Rep 2004;11:47-50.

28 Fukunaga M: Expression of D2-40 in lymphatic endothelium of normal tissues and in vascular tumours. Histopathology 2005;46: 396-402.

29 Choi WW, Lewis MM, Lawson D, et al: Angiogenic and lymphangiogenic microvessel density in breast carcinoma: correlation with clinicopathologic parameters and VEGFfamily gene expression. Mod Pathol 2005; 18: 143-152.

30 Giorgadze TA, Baloch ZW, Pasha T, Zhang PJ, Livolsi VA: Lymphatic and blood vessel density in the follicular patterned lesions of thyroid. Mod Pathol 2005;18:1424-1431.

31 Zeng Y, Opeskin K, Horvath LG, Sutherland RL, Williams ED: Lymphatic vessel density and lymph node metastasis in prostate cancer. Prostate 2005;65:222-230.

32 Ueno H, Murphy J, Jass JR, Mochizuki H, Talbot IC: Tumour 'budding' as an index to estimate the potential of aggressiveness in rectal cancer. Histopathology 2002;40:127132.

33 Hase K, Shatney C, Johnson D, Trollope M, Vierra M: Prognostic value of tumor 'budding' in patients with colorectal cancer. Dis Colon Rectum 1993;36:627-635.

34 Hase K, Shatney CH, Mochizuki H, et al: Long-term results of curative resection of 'minimally invasive' colorectal cancer. Dis Colon Rectum 1995;38:19-26.

35 Tominaga K, Nakanishi Y, Nimura S, Yoshimura K, Sakai Y, Shimoda T: Predictive histopathologic factors for lymph node metastasis in patients with nonpedunculated submucosal invasive colorectal carcinoma. Dis Colon Rectum 2005;48:92-100. 